tional model systems and also calls for new investigations using human biological and epidemiologic data. The iterative use of human and animal studies will bring the most rapid progress toward enhanced diagnoses, interventions to improve clinical outcomes, and preventative strategies for human birth defects.

\section{Acknowledgments}

The ideas presented in this manuscript have benefited from many discussions with our colleagues, in particular Kaare Christensen, Michael Dixon, Peter Jezewski, Andrew Lidral, Mary Marazita, Max Muenke, Paul Romitti, and Alex Vieira. Nancy Davin and Susie McConnell provided critical administrative support. This work was supported by NIH grants to J.C. Murray (DE08559) and B.C. Schutte (DE13513).

Address correspondence to: Jeffrey C. Murray, Department of Pediatrics and Craniofacial Anomalies Research Center, 2182 MedLabs, University of Iowa, Iowa City, Iowa 52242, USA. Phone: (319) 335-6897; Fax: (319) 3356970; E-mail: jeff-murray@uiowa.edu.
1. Murray, J.C. 2002. Gene/environment causes of cleft lip and/or palate. Clin. Genet. 61:248-256.

2. Rice, R., et al. 2004. Disruption of Fgf10/Fgfr $2 b$ coordinated epithelial-mesenchymal interactions causes cleft palate. J. Clin. Invest. 113:1692-1700. doi:10.1172/JCI200420384.

3. Satokata, I., and Maas, R. 1994. Msx 1 deficient mice exhibit cleft palate and abnormalities of craniofacial and tooth development. Nat. Genet. 6:348-356.

4. Proetzel, G., et al. 1995. Transforming growth factor beta 3 is required for second palate fusion. Nat. Genet. 11:409-414.

5. Jezewski, P., et al. 2003. Complete sequencing demonstrates a role for MSX1 in nonsyndromic cleft lip and palate. J. Med. Genet. 40:399-407.

6. Kondo, S., et al. 2002. Mutations in IRF6 cause Van der Woude and popliteal pterygium syndromes. Nat. Genet. 32:285-289.

7. Zucchero, T.M., et al. 2004. Interferon regulatory factor 6 (IRF6) is a modifier for isolated cleft lip and palate. N. Engl. J. Med. In press.

8. Dode, C., et al. 2003. Loss of function mutations in FGFR1 cause autosomal dominant Kallmann syndrome. Nat. Genet. 33:463-465.

9. Fitzpatrick, D.R., et al. 2003. Identification of SATB2 as the cleft palate gene on 2q32-q33. Hum. Mol. Genet. 12:2491-2501.

10. Zhang, Z., et al. 2002. Rescue of cleft palate in Msx1-deficient mice by transgenic Bmp4 reveals a network of BMP and Shh signaling in the regulation of mammalian palatogenesis. Development. 129:4135-4146.

11. Ornitz, D.M., and Itoh, N. 2001. Fibroblast growth factors. Genome Biol. 2:reviews3005.1-3005.12.

12. Braybrook, C., et al. 2002. Craniofacial expression of human and murine TBX22 correlates with the cleft palate and ankyloglossia phenotype observed in CPX patients. Hum. Mol. Genet. 11:2793-2804.

13. Mimura, J., et al. 1997. Loss of teratogenic response to 2,3,7,8-tetrachlorodinbenzo-p-dioxin (TCDD) in mice lacking the $\mathrm{Ah}$ (dioxin) receptor. Genes Cells. 2:645-654.

14. Dudas, M., Nagy, A., Laping, N.J., Moustakeas, A., and Kaartinen, V. 2004. Tgf- $\beta 3$-induced palatal fusion is mediated by Alk-5/Smad pathway. Dev. Biol. 266:96-108.

15. Hagiwara, N., Katarova, Z., Siracusa, L.D., and Brilliant, M.H. 2003. Nonneuronal expression of the GABA(A) beta3 subunit gene is required for normal palate development in mice. Dev. Biol. 254:93-101.

16. Little J., Cardy A., and Munger, R.G. 2004. Tobacco smoking and oral clefts: a meta-analysis. Bull. World Health Organ. 82:213-218.

17. Hayes, C. 2002. Environmental risk factors and oral clefts. In Cleft lip o palate from origin to treatment. D.F. Wyszynski, editor. Oxford University Press. Oxford, United Kingdom. 159-169.

18. Suzuki, K., et al. 2000. Mutations of PVRL1, encoding a cell-cell adhesion molecule/herpesvirus receptor, in cleft lip/palate-ectodermal dysplasia. Nat. Genet. 25:427-430.

19. Centers for Disease Control and Prevention (CDC). 2004. Spina bifida and anencephaly before and after folic acid mandate--United States, 1995-1996 and 1999-2000. MMWR Morb. Mortal. Wkly. Rep. 53:362-365.

20. Edison, R.J., and Muenke, M. 2004. Central nervous system and limb anomalies in case reports of first-trimester statin exposure. N. Engl. J. Med. 350:1579-1582.

\title{
Poliovirus proves IRES-istible in vivo
}

\author{
Bert L. Semler
}

Department of Microbiology and Molecular Genetics, College of Medicine, University of California, Irvine, California, USA.

\begin{abstract}
The genetic basis for the attenuation of polio vaccines has been known since the 1980s. Changes in the internal ribosome entry site, within the $5^{\prime}$ noncoding region of genomic RNAs, were presumed to reduce translation in certain target organs, leading to the conclusion that attenuation is mediated at the level of translation. A report in this issue of the JCI reveals that poliovirus tropism is, in part, determined after internal ribosome entry (see the related article beginning on page 1743).
\end{abstract}

The attenuation of poliovirus, the prototype member of the virus family Picornaviridae, by Albert Sabin's multiple, alternating passages of all three serotypes in different cultured cells and host animals led to the isolation of the vaccine strains now in use throughout much of the world. The nature of the genetic differences among the attenuated (avirulent) iso-

Nonstandard abbreviations used: coxsackievirus B (CVB); hepatitis C virus (HCV); internal ribosome entry site (IRES); noncoding region (NCR).

Conflict of interest: The author has declared that no conflict of interest exists.

Citation for this article:

J. Clin. Invest. 113:1678-1681 (2004).

doi:10.1172/JCI200422139. lates of poliovirus was unknown until the first complete genomic sequences of viral RNA were determined (1-4) and compared with the sequences of different Sabin strain isolates (4-6). Depending on the serotype, there were nucleotide differences scattered throughout the genome or concentrated in just a few loci in the Sabin strain viral RNAs. As discussed in the comprehensive review of poliovirus genetics by Wimmer and colleagues (7), the most functionally significant differences contributing to the attenuation phenotype of the Sabin vaccine strains of poliovirus were found in the genomic RNA sequences corresponding to the $5^{\prime}$ noncoding region ( $5^{\prime} \mathrm{NCR}$ ) and to sequences that encoded the capsid proteins, which make up the icosahedral shell of this nonenveloped virus particle. The genome organization of poliovirus RNA is depicted in Figure 1, while Figure 2 displays the RNA secondary structure of the $5^{\prime} \mathrm{NCR}$.

A major determinant of neurovirulence for all three serotypes of poliovirus was identified in the stem-loop V region of the $5^{\prime} \mathrm{NCR}$, indicated by the star in Figure 2 (ref. 8; for recent reviews, see refs. 9, 10). This stem-loop structure was later shown to be part of the internal ribosome entry site (IRES) present in the $5^{\prime}$ NCRs of all picornavirus positivestrand genomic RNAs (Figure 2). IRES elements permit the internal binding of ribosomes via a non-canonical, cap-independent mechanism of translation initiation utilized by some viruses and even a limited number of eukaryotic cellular mRNAs. There is some evidence for cell type-specific, cell cycle-dependent, and even developmentally regulated translation initiation mediated by cellular IRES elements (11). Thus, it would certainly be reasonable to assume that viral 


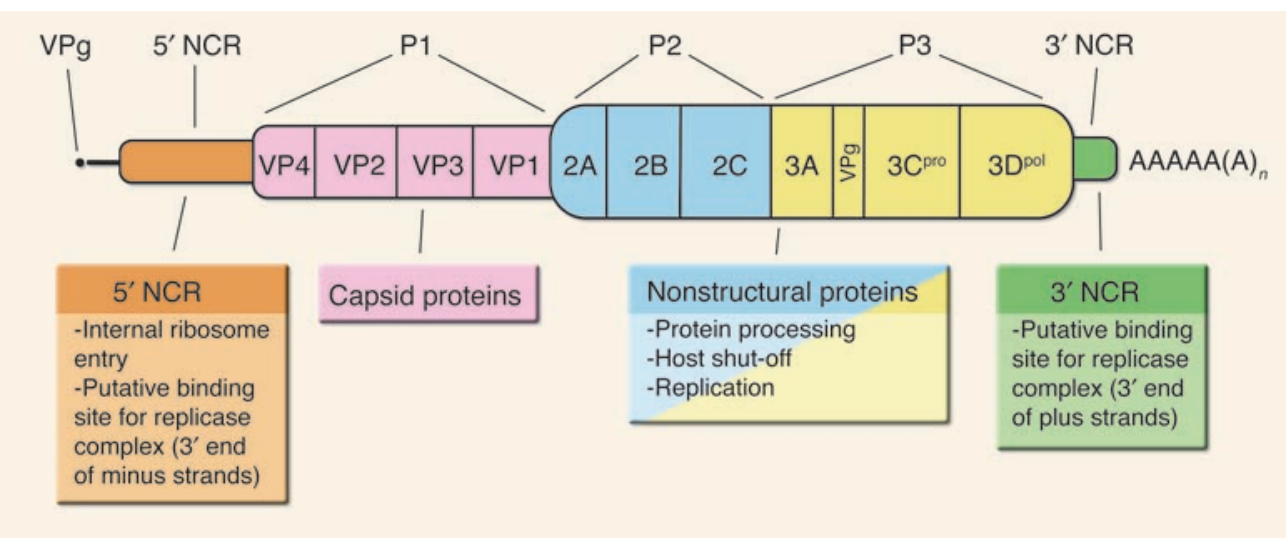

Figure 1

Functional map of the poliovirus genome. Genomic RNA is linked to a virus-encoded peptide $(\mathrm{VPg})$ at the $5^{\prime}$ end and a genetically coded poly(A) tract at the $3^{\prime}$ end. Viral RNA is depicted with a description of the functions of the various regions of the genome. The coding region of the virus is conventionally divided into three sections, referred to as $\mathrm{P} 1, \mathrm{P} 2$, and $\mathrm{P} 3$. The $\mathrm{P} 1$ region encodes the structural (capsid) proteins. The P2 region encodes proteins required for RNA replication and one of the viral proteinases responsible for host cell shut-off of cap-dependent translation. The $\mathrm{P} 3$ region encodes the major viral proteinase ( $3 \mathrm{C}^{\text {pro }}$ ), the viral RNA-dependent RNA polymerase (3Dpol), and other proteins required for RNA replication. The coding region is preceded by an unusually long $5^{\prime} \mathrm{NCR}$, which directs translation initiation by internal ribosome entry in the absence of cap-dependent functions. The viral genome also contains a short 3 ' NCR, which presumably contains cis-acting sequences involved in template recognition by the viral-replication initiation complex.

IRES elements, like the one encoded in the poliovirus genome, might have cell-specific requirements to achieve high levels of virusspecific translation initiation. Given the identification of a major attenuation determinant in an important RNA secondary structure embedded in the poliovirus IRES, this conclusion might appear foregone. Indeed, the analysis of attenuated and neurovirulent polioviruses using cell culture and in vitro translation assays provided considerable evidence for the role of the IRES in the biological properties of these viruses. RNA isolated from Sabin type 3 poliovirus was translated in vitro with reduced efficiency compared with RNAs from neurovirulent strains of type 3 poliovirus (12). The translation deficiency was later shown to be the result of the known attenuating mutation $(\mathrm{N} 472 \mathrm{C} \rightarrow \mathrm{U})$ in the IRES of the Sabin type 3 genome (13). A cell culture model using HeLa cells and a neuroblastoma cell line showed that recombinant viruses differing only by the $\mathrm{C} 472 \mathrm{U}$ mutation replicated with equal

\section{Figure 2}

Predicted RNA secondary structure of the poliovirus 5' NCR. Computer prediction and chemical and enzymatic RNA-structure probing were used to deduce a consensus RNA conformation. Conserved sequences among picornaviruses include a GNRA tetraloop (thought to function in tertiary interactions of RNAs and in protein binding), A/C-rich loops, and a pyrimidine-rich region just upstream of the conserved AUG codon. The IRES domain is boxed by red lines. efficiency in HeLa cells (14). However, infection of neuroblastoma cells with these two viruses showed that the virus containing the uridine residue at $\mathrm{N} 472$ grew to lower titers in these cells. The attenuated virus also had reduced translation efficiency during infection of the neuronal cell line, consistent with the above-noted in vitro translation results for Sabin 3 poliovirus RNAs. In addition, site-directed mutations that altered the RNA sequences and secondary structures adjacent to the major attenuation determinant in the $5^{\prime}$ NCR of type 1 poliovirus (N480) led to neuronal cell-specific growth defects in cul- tured cells and to in vitro translation defects in S-10 extracts derived from neuroblastoma cells grown in suspension culture (15). As they report in this issue of the JCI, Kauder and Racaniello formally tested the hypothesis that IRES-mediated translation initiation is a major determinant of poliovirus tropism and pathogenesis (16).

\section{Transgenic mouse models of poliovirus pathogenesis}

A major advance in the study of poliovirus attenuation/neurovirulence determinants was reported by Racaniello and colleagues

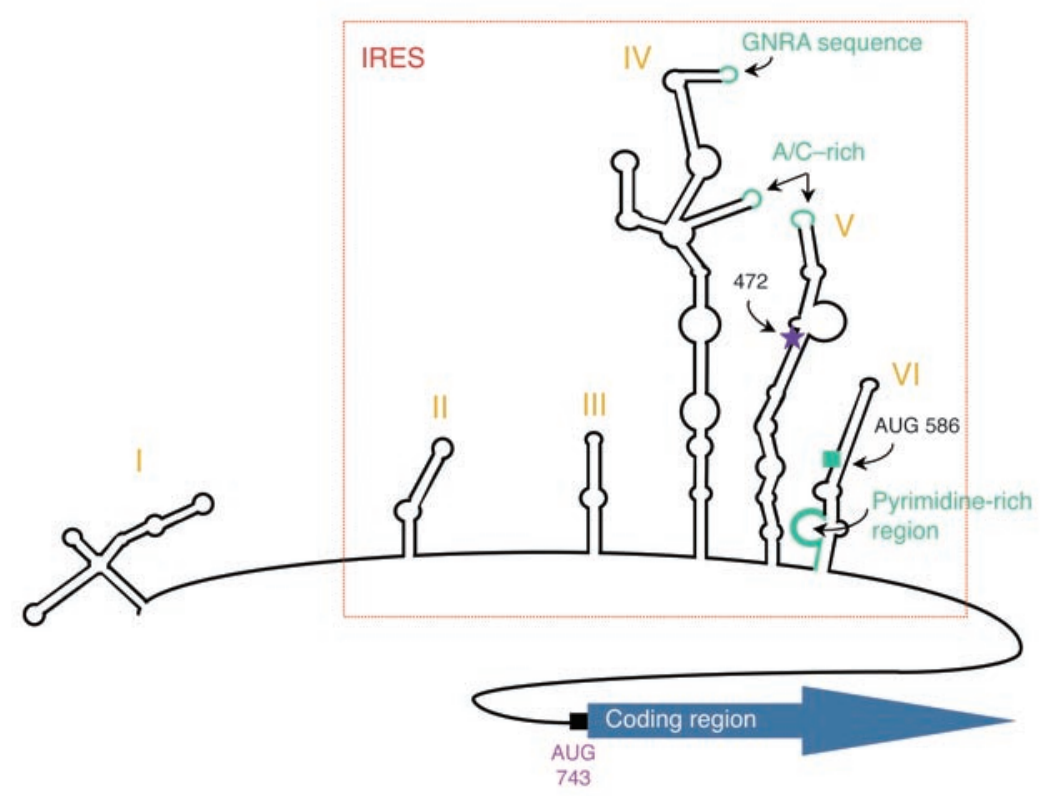


and by Nomoto and colleagues when these investigators engineered transgenic mice expressing the receptor for poliovirus from human cells $(17,18)$. These mice were susceptible to poliovirus infection and could produce disease symptoms and paralysis similar to those produced in primates. Use of transgenic mice for poliovirus-pathogenesis studies has obvious practical advantages over the much more cumbersome and expensive use of monkeys. Moreover, viruses with lesions known to attenuate poliovirus neurovirulence in monkeys also proved to be attenuated in the transgenic mouse model $(17,18)$. Tissue-specific poliovirus replication in brain, spinal cord, and skeletal muscle was demonstrated in inoculated transgenic mice despite the expression of the human poliovirus receptor in a large number of tissue types, suggesting that receptor expression was necessary but not sufficient for disease progression (19). These results and those derived from in vitro and cell culture approaches (discussed above), coupled with the original report of poliovirus binding to tissue homogenates from organs normally not susceptible to poliovirus infection (20), led to the pursuit of post-entry determinants of poliovirus pathogenesis using the transgenic mouse model.

Using a chimeric virus containing a replacement of IRES sequences in the poliovirus type $15^{\prime} \mathrm{NCR}$ with the corresponding sequences from human rhinovirus type 2, Gromeier and colleagues showed that the chimeric virus grew to lower titers in cultured neuroblastoma cells than in cells of non-neuronal origin and was attenuated for neurovirulence in transgenic mice expressing the poliovirus receptor (21). Additional studies confirmed the validity of these findings following inoculation of cynomolgus monkeys and suggested that interactions between specific stem-loop structures (V and VI) within the poliovirus IRES are a major determinant of neurovirulence in addition to the known attenuating lesion in stem-loop V (22). Recently, Nomoto and colleagues reported altered tissuespecific viral replication (in the transgenic mouse model) of a chimeric poliovirus that contains the IRES sequences from hepatitis $\mathrm{C}$ virus ( $\mathrm{HCV}$ ), further emphasizing the importance of these RNA sequences in poliovirus tropism and attenuation (23).

\section{Novel recombinant viruses}

In the elegant and comprehensive study described in their article, Kauder and Racaniello used bicistronic reporter genes to demonstrate that tissue-specific expression of reporter mRNA translation did not co-vary when the IRES was changed from that of poliovirus to that of coxsackievirus $\mathrm{B}$ (CVB) or HCV (16). What is novel about the present studies is the use of a recombinant adenovirus to deliver the reporter constructs to different tissues in the infected mice, a process that is independent of normal poliovirus-receptor interactions. This resulted in the infection of cells from a broad distribution of mouse tissues that would allow the assessment of IRES activity in cells that normally support poliovirus replication as well as those that don't. The authors carried out important controls to show that they were truly measuring IRESdependent translation and not an artifact of bicistronic constructs (e.g., altered RNA stability). They then went on to generate recombinant polioviruses containing the IRES substitutions used in their reporter assays. These viruses were used to infect mice transgenic for the poliovirus receptor. Despite the presence of the CVB or HCV IRES elements, the recombinant polioviruses retained the wild-type tropism for brain and spinal cord. The authors also showed that a single point mutation in the IRES (C472U), thought to be a major determinant of poliovirus attenuation of neurovirulence, still allowed replication in the brains and spinal cords of newborn transgenic mice but not in adult transgenic mice. Importantly, Kauder and Racaniello demonstrated that the populations of viruses maintained their genetic homogeneity (containing the mutated or wild-type genomic sequence at the single locus in the viral IRES) after replication in mice, ruling out early reversion events as the source of the unexpected neurovirulence phenotypes.

The paper by Kauder and Racaniello describes novel and provocative results regarding the genetic determinants of poliovirus attenuation in transgenic mice (16). Its conclusions differ from those derived from previous cell culture and in vitro studies, perhaps because of the limitations of system integrity inherent in any in vitro or ex vivo analysis. For example, extracts made from neuronal cells or cells derived from neuronal tumors may indeed have reduced concentrations of a factor(s) required for IRES-mediated translation of poliovirus RNA. However, this factor may not be limiting in intact tissues or may not participate in a rate-limiting step in the progression to cell-specific disease. In addition, cultures of infected neuronal cells that show reduced levels of viral proteins produced by isolates of attenuated poliovirus may mask the real defect in RNA replication, which would ultimately produce fewer progeny RNAs available for additional rounds of translation and genome amplification.

\section{Beyond the translation paradigm}

The data reported by Kauder and Racaniello have provided an important challenge to existing paradigms for the putative mechanisms of poliovirus neurovirulence and attenuation (16). Such mechanisms have been thought to operate primarily at the level of translation initiation through IRES functions in a specific manner in cells of neuronal origin. Although viral replication is clearly tissue- and cell-specific (as confirmed in the current studies), Kauder and Racaniello's data suggest that the intracellular stage of the virus life cycle at which this specificity is exhibited occurs after the stage of translation initiation mediated by the poliovirus IRES. Their data may point to tissue-specific steps in viral RNA replication that have novel requirements for sequences contained within the IRES. RNA replication determinants within the IRES of poliovirus RNAs have been previously identified $(24,25)$, so there is a genetic precedent for posttranslation determinants of viral replication embedded within these complex RNA elements. Uncoupling the translation functions from the RNA replication functions affected by specific RNA sequences remains a complex experimental task, especially given the clear differences seen when poliovirus replication is studied in cultured cells compared with outcomes measured in infected transgenic mice. As Kauder and Racaniello point out, even such highly valuable experimental tools as transgenic mice expressing the poliovirus receptor have their limitations. In failing to recapitulate the alimentary tract route of infection, the current transgenic mouse model may be unable to explain the age-dependent differences in poliovirus pathogenesis seen in the present study. Recent studies have reported new lines of transgenic mice that are susceptible to poliovirus infection via mucosal routes $(26,27)$, suggesting the possibility of further studies of poliovirus attenuation/ neurovirulence determinants in small-animal models via more relevant routes of infection. It remains to be seen whether ongoing and future mechanistic studies of poliovirus attenuation and neurovirulence using transgenic mice and monkey model systems will be completed prior to the cessation of research with infectious poliovirus that the 
World Health Organization's plan to eradicate poliovirus from the planet will necessitate. Given some of the ongoing logistical problems with this noble effort, researchers may yet have time to uncover some of the long-standing mysteries of viral pathogenesis presented by the unique tropism and disease characteristics of poliovirus infections.

Address correspondence to: Bert L. Semler, Department of Microbiology and Molecular Genetics, College of Medicine, Medical Sciences B240, University of California, Irvine, California 92697, USA. Phone: (949) 824-7573; Fax: (949) 824-2694; E-mail: blsemler@uci.edu.

1. Kitamura, N., et al. 1981. Primary structure, gene organization and polypeptide expression of poliovirus RNA. Nature. 291:547-553.

2. Racaniello, V.R., and Baltimore, D. 1981. Molecular cloning of poliovirus cDNA and determination of the complete nucleotide sequence of the viral genome. Proc. Natl. Acad. Sci. U. S. A. 78:4887-4891.

3. Stanway, G., et al. 1983. The nucleotide sequence of poliovirus type 3 leon $12 \mathrm{a} 1 \mathrm{~b}$ : comparison with poliovirus type 1. Nucleic Acids Res. 11:5629-5643.

4. Toyoda, H., et al. 1984. Complete nucleotide sequences of all three poliovirus serotype genomes. Implication for genetic relationship, gene function and antigenic determinants. J. Mol. Biol. 174:561-585.

5. Nomoto, A., et al. 1982. Complete nucleotidesequence of the attenuated poliovirus Sabin 1 strain genome. Proc. Natl. Acad. Sci. U. S. A. 79:5793-5797.

6. Stanway, G., et al. 1984. Comparison of the complete nucleotide sequences of the genomes of the neurovirulent poliovirus $\mathrm{P} 3 / \mathrm{Leon} / 37$ and its attenuated Sabin vaccine derivative P3/Leon 12a1b. Proc. Natl. Acad. Sci. U. S. A. 81:1539-1543.

7. Wimmer, E., Hellen, C.U., and Cao, X. 1993. Genetics of poliovirus. Annu. Rev. Genet. 27:353-436.

8. Evans, D.M.A., et al. 1985. Increased neurovirulence associated with a single nucleotide change in a noncoding region of the Sabin type 3 poliovaccine genome. Nature. 314:548-550.

9. Gromeier, M., and Nomoto, A. 2002. Determinants of poliovirus pathogenesis. In Molecular biology of picornaviruses. B.L. Semler and E. Wimmer, editors. ASM Press. Washington, DC, USA. 367-379.

10. Minor, P.D., and Almond, J. 2002. Poliovirus vaccines: molecular biology and immune response. In Molecular biology of picornaviruses. B.L. Semler and E. Wimmer, editors. ASM Press. Washington, DC, USA. 381-390.

11. Hellen, C.U., and Sarnow, P. 2001. Internal ribosome entry sites in eukaryotic mRNA molecules. Genes Dev. 15:1593-1612.

12. Svitkin, Y.V., Maslova, S.V., and Agol, V.I. 1985. The genomes of attenuated and virulent poliovirus strains differ in their in vitro translation efficiencies. Virology. 147:243-252.

13. Svitkin, Y.V., Cammack, N., Minor, P.D., and Almond, J.W. 1990. Translation deficiency of the Sabin type 3 poliovirus genome: association with an attenuating mutation C472 $\rightarrow$ U. Virology. 175:103-109.

14. La Monica, N., and Racaniello, V.R. 1989. Differences in replication of attenuated and neurovirulent polioviruses in human neuroblastoma cell line SH-SY5Y. J. Virol. 63:2357-2360.

15. Haller, A.A., Stewart, S.R., and Semler, B.L. 1996 Attenuation stem-loop lesions in the 5 ' noncoding region of poliovirus RNA: neuronal cell-specific translation defects. J. Virol. 70:1467-1474.

16. Kauder, S.E., and Racaniello, V.R. 2004. Poliovirus tropism and attenuation are determined after internal ribosome entry. J. Clin. Invest. 113:1743-1753. doi:101172/JCI200421323.
17. Ren, R.B., Costantini, F., Gorgacz, E.J., Lee, J.J., and Racaniello, V.R. 1990. Transgenic mice expressing a human poliovirus receptor: a new model for poliomyelitis. Cell. 63:353-362.

18. Koike, S., et al. 1991. Transgenic mice susceptible to poliovirus. Proc. Natl. Acad. Sci. U. S. A. 88:951-955.

19. Ren, R., and Racaniello, V.R. 1992. Human poliovirus receptor gene expression and poliovirus tissue tropism in transgenic mice. J. Virol. 66:296-304.

20. Holland, J.J. 1961. Receptor affinities as major determinants of enterovirus tissue tropisms in humans. Virology. 15:312-326.

21. Gromeier, M., Alexander, L., and Wimmer, E. 1996. Internal ribosomal entry site substitution eliminates neurovirulence in intergeneric poliovirus recombinants. Proc. Natl. Acad. Sci. U. S. A. 93:2370-2375.

22. Gromeier, M., Bossert, B., Arita, M., Nomoto, A., and Wimmer, E. 1999. Dual stem loops within the poliovirus internal ribosomal entry site control neurovirulence. J. Virol. 73:958-964.

23. Yanagiya, A., et al. 2003. Tissue-specific replicating capacity of a chimeric poliovirus that carries the internal ribosome entry site of hepatitis $\mathrm{C}$ virus in a new mouse model transgenic for the human poliovirus receptor. J. Virol. 77:10479-10487.

24. Borman, A.M., Deliat, F.G., and Kean, K.M. 1994. Sequences within the poliovirus internal ribosome entry segment control viral RNA synthesis. $E M B O$ J. 13:3149-3157.

25. Shiroki, K., et al. 1995. A new cis-acting element for RNA replication within the $5^{\prime}$ noncoding region of poliovirus type 1 RNA. J. Virol. 69:6825-6832.

26. Crotty, S., Hix, L., Sigal, L.J., and Andino, R. 2002. Poliovirus pathogenesis in a new poliovirus receptor transgenic mouse model: age-dependent paralysis and a mucosal route of infection. J. Gen. Virol. 83:1707-1720

27. Nagata, N., et al. 2004. A poliomyelitis model through mucosal infection in transgenic mice bearing human poliovirus receptor, TgPVR21. Virology. 321:87-100.

\title{
Is transplantation tolerable?
}

\author{
Terry B. Strom
}

Division of Immunology and Transplant Research Center, Beth Israel Deaconess Medical Center, Harvard Medical School, Boston, Massachusetts, USA.

\begin{abstract}
To test the hypothesis that chronic stimulation of $\mathrm{T}$ cells with a weak agonistic antigen will generate regulatory $T$ cells and immune tolerance, a study reported in this issue (see the related article beginning on page 1754) employed the redesign of a minor histocompatibility antigen. Using knowledge of residues at which the antigen contacts the $T$ cell receptor, a weak agonist was produced. Pretreatment with this altered antigen produced transplant tolerance, generation of regulatory $T$ cells, and a loss of many antigen-reactive $T$ cells.
\end{abstract}

In brackish waters once trafficked by oldschool cellular immunologists, precious texts describing the wonders of somewhat

Nonstandard abbreviations used: altered peptide ligand (APL); glucocorticoid-induced TNF receptor (GITR); T cell receptor (TCR).

Conflict of interest: The author has declared that no conflict of interest exists.

Citation for this article:

J. Clin. Invest. 113:1681-1683 (2004).

doi:10.1172/JCI200422153. ill-defined suppressor lymphocytes (1) were jettisoned. Later works, also discarded, described lymphocytes able to protect rather than destroy foreign tissues in adult hosts rendered tolerant to organ transplants $(2-4)$. CD4 ${ }^{+}$, IL-2 receptor-positive $\left(\mathrm{CD} 25^{+}\right) \mathrm{T}$ cells capable of countering the graft-destroying properties of alloaggressive $\mathrm{T}$ cells were identified by Hall and his colleagues in rodent transplant models (2). Nonetheless, following the fashion of the time, many of us cleansed our grants and manuscripts of any mention of suppressor $\mathrm{T}$ cells. Following a revival of interest in suppressor, or regulatory, $\mathrm{T}$ cells, Chen et al. in this issue of the JCI (5) have now redesigned a minor histocompatibility antigen to test the hypothesis that chronic stimulation of $\mathrm{T}$ cells with a weak agonistic antigen will generate regulatory $T$ cells and produce immune tolerance.

In the 1990s, an era in which the phrase "suppressor T cells" was uttered only in hushed tones, a series of brilliant experiments by Waldmann $(6,7)$ and his colleagues identified a crucial graft-protecting $\mathrm{T}$ cell-dependent network in hosts rendered tolerant to transplants by means other than creation of total and enduring deletion of antidonor clones. Tolerant host 